\title{
ADMINISTRATION OF ETHER.
}

\author{
By \\ J. H. T. Challis, D.A. \\ Anaesthetist, London Hospital.
}

Ether vapour has stood the test of one hundred years this year and it still holds first place as the safest anaesthetic agent. It has only two indisputable drawbacks in comparison with all other agents; these are inflammability and the dislike most people have for its smell. All other disadvantages so often lauded are by no means proven. It is not suggested for a moment that ether is the first and last word in anaesthesia but until such time as a safer and better agent or agents are discovered, it is well to be conversant with the various methods of its administration.

These are:-

I. Intravenously in a 5 per cent solution in normal saline.

2. Rectally.

3. Intravenously in combination with other intravenous anaesthetics such as pentothal sodium.

4. By inhalation.

The intravenous injection of ether in saline and the rectal administration of it has become obsolete but the addition of io to 20 c.c. of ether to 450 c.c. of saline or glucose with 2 gms. of pentothal sodium certainly greatly adds to the smoothness and ease of administration of this type of intravenous anaesthesia.

The methods of inhalation of ether anaesthesia are exceedingly numerous but only those in present-day practical use will be touched upon.

I. Open ether on a Schimmelbusch mask. This method of induction and maintenance, in very small children, in the profoundly shocked, and where apparatus is not available, may still be fully justified and certainly any anaesthetist must be capable of using this method. The induction is done slowly on a mask covered with twelve to fourteen single layers of gauze and with a pad of gamgee covering the face and eyes. In the initial stages adequate air or oxygen is allowed between the mask and gamgee. Gradually, as the strength of the ether vapour is increased, the face piece is brought into contact with the gamgee. In small children breath holding and cyanosis are apt to occur. A mixture of 5 per cent carbon dioxide in 95 per cent oxygen is invaluable at this stage of the induction, if trickled in slowly under the mask. The carbon dioxide should be discontinued immediately a regular automatic respiratory excursion is established. In the shocked patient, little difficulty will be encountered during induction $\frac{\overline{ }}{\bar{n}}$ but the establishment of surgical anaesthesia is $\vec{\nabla}$

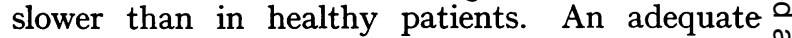
supply of oxygen does much to improve the poor $\infty$ condition of these cases. In really bad anaes- $\overrightarrow{0}$ thetic risks such refinements as Pentothal Sodium $\overrightarrow{-}$ or even the addition of chloroform to ether are $\vec{\omega}$ very liable to produce sudden death.

2. Induction with ethyl chloride and maintenance with ether. This method certainly has the advantage of speed, but the change from the one to $N$ the other agent is not without its difficulties since $N$ if the ethyl. chloride induction is pushed slightly $N$ too far, laryngeal and masseter spasm is very $\vec{\omega}$ liable to occur and trouble will be experienced in 을 re-establishing an airway, or conversely if the change is slightly early patients are apt to recover $\vec{o}$ to the fighting stage before sufficient ether has been inhaled to establish smooth anaesthesia Again a mixture of 5 per cent carbon dioxide in 95 per cent oxygen facilitates the change oven $\overrightarrow{0}$ The ethyl chloride-ether combination is not to unpleasant and is usually preferred to the slowe⿳亠丷厂 and laboured administration of mixtures of chloroform and ether and it is suitable especially for short surgical procedures where nitrous oxide or ethyl chloride alone give insufficient operating time, and, of course, when more refined methods are not possible.

3. Inductions with chloroform and ether mixtures and maintenance with ether. Teaching varies as to the quantity of chloroform used; it may be from equal parts to as little as one part chloroform to twelve parts ether. The commonest mixture is one part chloroform to two parts ether. The mixture must be freshly prepared since with keeping, chemical change takes place with the giving off of heat. Therefore, the mixture must be made up immediately before use. The induction should be smooth and the change over to an open $\frac{D}{0}$ ether easy. If full anaesthesia is established before this change is attempted it is as well to $N$ use a fresh mask when going from the mixture to plain ether, as residual chloroform may collect 0 on the old mask, and the stimulating effect of the $\omega$ ether vapour may cause deeper respirations to occur and in this way an overdose of this chloroform 0 residue to be inhaled, causing sudden death. This method of anaesthesia is antiquated and now has? little to recommend it except the non-availability of other methods. 
4. Induction with nitrous oxide-oxygen and followed with ether.

There are three methods of doing this:

(a). With a Clovers inhaler or other like apparatus which are now considered obsolete.

(b). Semi-closed method using any type of Boyles machine.The induction is done with nitrous oxide and ether added as soon as anaesthesia is established: then sufficient oxygen is turned on to maintain good oxygenation and ether is gradually introduced until the correct plane of anaesthesia is reached. The amount required for abdominal relaxation is considerable. Many anaesthetists unfortunately sometimes omit to mention they are giving ether, and refer to it as gas and oxygen, thus giving an erroneous idea of the capabilities of pure gas and oxygen.

The addition of ether to nitrous oxide-oxygen requires careful management, otherwise coughing and laryngeal spasm will occur. These difficulties are much less marked when the patient has been premedicated with omnopon gr. I/3 and scopolamine gr. $1 / 150$. (This is the normal dose for healthy average adults but should be halved in the aged and feeble and replaced by other drugs in children.) The administration of sufficient pentothal sodium intravenously should certainly not interfere with this method of anaesthesia. Once again the addition of small quantities of carbon dioxide for a short time immediately before and during the first half dozen breaths of ether will greatly facilitate the ease and speed of establishing full anaesthesia.

5. Closed ether. Once full anaesthesia has been established by any recognised method, usually the nitrous oxide-oxygen and ether sequence, the nitrous oxide is turned off and oxygen reduced to the basic amount required, which varies slightly in each individual, depending upon the varying physical and physiological conditions. Usually 250 c.c. to 350 c.c. per minute is a good average. The carbon dioxide is absorbed by soda lime and the patient breathes completely or partially over ether. Thus more and more ether is vaporised until the required depth of anaesthesia is reached, when theoretically the ether can be turned off and the anaesthetic plane should remain at a constant depth. But in actual practice, probably by loss from skin and mechanical imperfections, it is always necessary to add periodically fresh supplies of ether or to continuously allow a small percentage of the patient's breath to pass over the ether so as to replace this unavoidable loss. Once a closed circuit ether anaesthesia has been fully established it is highly satisfactory. Respiration is quiet and shallow owing to the partial absorption of $\frac{3}{\mathbb{D}}$ the stimulating agent carbon dioxide. Small $\frac{\Omega}{C}$ variations of depth are quickly and easily adjusted $\stackrel{c}{\Rightarrow}$ by altering the amount of the patient's respirations $\vec{c}$ ? passing over the ether. The loss of heat and $\frac{7}{0}$ moisture by expired air is diminished as the $\frac{0}{0}$ patient rebreathes the same mixture over and $\bar{\omega}$. over again and the heat given off by the soda $\widehat{\Phi}$ lime helps to keep the temperature constant. $\frac{2}{2}$ The disadvantages of this method are that the ${ }^{\infty}$ apparatus is heavy, clumsy and expensive but $\overrightarrow{0}$ the economy in ether, nitrous oxide and oxygen $\overrightarrow{-}$ will soon counter-balance the initial expense of $\vec{\sigma}$ the machine if it is used frequently.

Ether is the only inhalation anaesthetic which $\frac{0}{3}$ if properly administered will produce complete muscular relaxation within normal limits of $N$ safety. Cyclopropane certainly is not dependable ô in Ioo per cent of cases and chloroform is not $\stackrel{N}{\omega}$ without danger when the deeper stages of anaes- $\frac{\omega}{\omega}$ thesia are required. Ether is usually blamed for 0 all post-operative lung complications, this is not $\stackrel{5}{\rightarrow}$ supported by fact; many cases suffering from 0 lung complaints appear to tolerate ether anaes- $\frac{?}{0}$ thesia better than cyclopropane or nitrous oxide $\frac{0}{0}$ Another failing of ether is said to be post-operative coughing and vomiting. Granted that at the $\overrightarrow{0}$ time it is most unpleasant for the patient it is the long run a great advantage that the patier empties his chest and perhaps his stomach of $\frac{?}{\xi}$ undesirable contents and in doing so is forced to take large breaths thus expanding his lungs after ${ }^{2}$ lying flat for perhaps two to three hours on the $\frac{\alpha}{\infty}$ operating table. With the more modern anaes- $\stackrel{2}{\overrightarrow{2}}$ thetics (pentothal, cyclopropane, etc.) there is a tendency for the patients to return to their beds and remain quiet for many hours after their operation with shallow undisturbed respiration. During this time all sorts of corruptions are possibly $\frac{0}{3}$ taking place, and having no stimulus to cough or expectorate, plugs of mucus remain undisturbed 3 . and it may be hours before the lungs are properly and fully expanded. Even chest anaesthetists $\stackrel{\rho}{\xi}$ when having difficulty in establishing a smooth, $ᄋ$ even respiration or before the passage of bronchial 5 or intratracheal tubes, add a judicious amount $\frac{7}{0}$ of ether vapour in any operation. Should the patient's condition flag, it is remarkable to see $\widetilde{\sim}$ the improvement that follows the addition of ether $\sim$ vapour to the patient's colour, respiration and pulse.

The consumption of anaesthetic ether in an average London teaching hospital is a half to three quarters of a ton per annum. This fact $\mathbb{\Phi}_{0}$ may help to disillusion those ultra-modernists? who believe that cyclopropane, pentothal, sodium $\frac{T}{0}$ and curarine are the last words in anaesthesia. 